\title{
ON PEOPLE AND COMPLEXITY IN HEALTHCARE SERVICE SUPPLY
}

\author{
PER ENGELSETH ${ }^{1} \&$ BRIAN E. WHITE ${ }^{2}$ \\ ${ }^{1}$ Department of Logistics, Molde University College, Norway. \\ ${ }^{2}$ Complexity Are Us $\leftarrow$ Systems Engineering Strategies, USA.
}

\begin{abstract}
Healthcare logistics is treated as a fundamentally emergent complex system primarily because a broad range of stakeholders are included. The patient is the primary "customer" of the service producers in the supply chain, including the doctors, nurses, medicine and insurance providers, and hospital administrators. Problematic issues regarding healthcare quality that need to be solved or improved are discussed, and suggestions for furthering and accelerating progress are offered. Careful application of information technology in designing appropriate information systems is advocated. Three specific illustrative cases of healthcare services that have been analyzed and assessed are summarized. The overall intent is to motivate creative processes for delivering more efficient and effective healthcare utilizing complex system behaviors and engineering principles, and an ethically-founded worldview.

Keywords: case studies, collaboration, complex systems engineering, complex systems, ecosystems, healthcare services, information systems, interdependencies, logistics, process emergence, supply chain management
\end{abstract}

\section{INTRODUCTION}

Healthcare logistical support is crucial in enabling overall service quality. Clearly, there are shortcomings in this and other areas, especially in the US, where healthcare expenditures represent about $20 \%$ of the Gross National Product (GNP). In addressing these issues here, we consider people problems as the most critical but we are also interested in healthcare management, and how best to apply information technology (IT) in creating information systems (ISs) that can make a positive difference.

Primarily because of the intensive composition of people, e.g., patients and care providers, in particular, understand that healthcare services are a very complex system. One of the key tenets in dealing with a complex system is the use of stakeholder analysis to help form the basis for actions in identifying and analyzing problems, and formulating potential solutions to be explored and implemented, perhaps on a trial basis to learn whether they help or not. The interactions of people both within and outside their organizations need to be studied, characterized, and revamped, as necessary. In a supply chain producers and customers are both value-creating and sensemaking agents that impact healthcare quality.

Behaviors of complex systems, and complex systems engineering principles [1] are cited throughout to help emphasize and generalize some of the specific points and comments of this paper. The overall hope is to raise awareness of the more important issues and to help stir concern and follow-up action to improve healthcare delivery and outcomes.

\section{BACKGROUND}

In this section some previous and apropos work in the fields of complexity, systems thinking, and complex systems engineering are cited. Healthcare problems and potential pathways to solutions are also discussed. 


\subsection{Previous Work}

The nature of complex systems and suggestions for complex systems engineering are described in many publications, e.g, [2-9]. In perusing the literature on these topics, one soon realizes that the people associated with the system often generate a high degree of complexity; and that intentionally including such stakeholders in the system to be engineered is necessary for creating more viable pathways toward system development and improvement. Nevertheless, good, available, up-to-date, and tested technology is often crucial in helping to solve human-centric complex problems. We propose to apply IT in establishing ISs amenable to effective healthcare logistical supply chain capabilities.

IT's resource base includes integrated information hardware and software design, development, and use. Three aspects of IS potential are discussed in [10]: (1) features of connectivity associated with people and documentation; (2) aspects of big data (BD); and (3) application of IT to integrate multi-tier service supply chains. Organizing such information, focusing on agency in a complex system, and describing the tools used by people (primarily the agents) to reach system goals seeks to produce supply chain value as the overarching system purpose. In addition, the ethical aspects of all three of these issues (connectivity, BD, and IT) should be considered. This is especially pertinent since healthcare concerns logistical services that directly impact human well-being.

\subsection{What's the Problem?}

Healthcare services need to be improved. Witness the prevailing indicators of exorbitant hospital, doctor, and drug costs, uninsured emergency room visits, and insufficient emphasis on preventative medicine, for example. Since people are embedded in organizing, operating, managing, and communicating within the logistical supply chain of healthcare, whatever improves the interactions of those people in providing healthcare services should positively affect overall performance. Later in this text we provide some narratives describing current actual standing of services in this industrial sector.

We deemphasize what seems to be a common mindset that technology alone can/will provide all the answers in enabling and delivering healthcare services. Adopting a people-centric "mindsight," [11] a term suggesting more open-minded flexibility than the rigidity-of-thought term, "mindset," seems more appropriate. Furthermore, viewing the healthcare providers as a highly interconnected and interactive network broadens the usual scope and expands the often too-narrow boundary of the system of interest.

Fundamentally, people are not only important in the production-and-supply side of healthcare, other supporting agents, people associated more with patients, for example, are the principal constituents of the healthcare problem side. The healthcare service providers and the related agents need to be concerned with not only: (1) how best to treat the sick efficiently; but also, better yet, (2) our overarching higher moral responsibility to make and keep everyone healthy more effectively. However, even then, individual mindsets/sights can be rather limited, and a healthy collaboration is required.

Healthcare services comprise a special industry with important particularities to grasp in order to develop them further. Interdependencies vary between different types of supply chains since they impact how people interact to produce a service. Turning now to the interactions of the people involved in the healthcare logistical supply chain, the nature of their interdependencies arises as an important issue. As discussed in detail in [10], 
interdependencies can range from sequential (serial), to reciprocal (two-way), to pooled (networked), where these three types are characterized as moving in the direction of increasing importance (value) in similarly more complex systems. To the extent one can design these interdependent relationships, the focus should be on networked interdependencies as the most general and potentially most beneficial, where little, if anything, will be omitted from consideration. However, after thorough analysis some networked interdependencies might be reduced to the reciprocal type without any loss of benefit but with a concomitant increase in effectiveness or efficiency. Similarly, upon further detailed analysis, a reciprocal interdependency might be simplified to a sequential type to achieve a net gain in effectiveness/ efficiency without any loss in benefit.

This is akin to saying that classical conventional, or traditional systems engineering techniques certainly can be usefully applied if the system at hand is not too complex. However, the more difficult systems, viz., system of systems (SoSs), enterprises, and the most complex systems require complementary techniques to bring the "requisite variety" [12] necessary to cope with the problems.

Network interactions are key in producing and delivering exemplar services in almost any domain but especially in healthcare where people are central players that comprise localized and extended networks spanning their concerns, whether conscious and intentional, or unconscious and intuitive. Through their actions, assisted with state-of-the-art technology to enable effective and efficient communications, healthcare givers and recipients engender a dynamic network of nodes and links, where the more influential can be represented by larger nodes with more incoming and outgoing links, akin to a "hub and spoke" supply chain.

The customers need to provide inputs (e.g., their identity, location, tangible resources, and health concerns) to, and feedback (e.g., their updated health status, degree of satisfaction, and further requests) about the healthcare process. Inadequate service quality results from, e.g., ad hoc, seemingly random, and delayed or duplicate message arrivals; their unclear content or inconsistent specifications; and errors in tracking current medical inventories, producing remedies, and implementing operational transactions. For more detail, cf., [10].

Providers are typically either unaware of or distracted from these difficulties. They tend to make insufficient efforts to mount systemic studies of the problems, achieve consensus among their collaborators, and commandeer resources necessary for significant improvements. Pooling different types of resources and configuring services in different ways increase service production complexity. Hospitalization involves a great deal of variation. For instance, a given ailment may be confronted differently even within the same hospital. This variation in service includes the numbers and types of patients in the reception ward, various healthcare personnel with different operational preferences, and the use of non-standard rooms and equipment, as well as time-line variations in capacity as impacted/constrained by other patient numbers, types of ailments, room availabilities, tools, medicines, health personnel, etc. [10].

\subsection{What's the Solution?}

Before proceeding it is important to understand or realize that system complexity is not necessarily a random chaotic thing out there. The behaviors of complex systems, most effectively operating at the edge of chaos, are not "random" or stochastic; they do not arise purely by chance. Such a "healthy" complex system continuously (or continually) evolves based on component interactions within its boundary and interactions with its environment across its 
boundary. Thankfully, a complex system can be influenced by design, or, more likely, thoughtful interventions that may, with sufficient time, lead to improved behaviors-or not; if the latter, one must consider taking a different approach and try to re-intervene [6].

What can one discover about how a complex system is responding to its interactions or our interventions? Typically, these responses are exhibited by patterns that may be observed but are often difficult to interpret. Simple, direct responses cannot often be reliably correlated with particular interactions or interventions. So, although there is cause for hope, the challenges are great. However, much can be learned about the patterns of a complex system by applying the tools of agent-based modeling and simulation in a virtual imitation of the complex system that operates in parallel with the original, perhaps in a speeded-up time frame [1]. We encourage researchers and workers in healthcare to pursue such approaches to help discover improved methodologies.

Healthcare logistics, with its inherent industrial particularities, may be effectively described and analyzed, for development as well as operational purposes, as a complex system supported by an IS. Complexity can be defined as: “... a property of an open system that consists of a large number of diverse, intelligent, partially anonymous, richly interconnected components, often called agents, [with] no centralized control and whose behavior emerges from the intricate interaction of agents and is therefore uncertain without being random" [13, p. 5]. These authors, Rzevski and Skobelev, also point out that the key features of complexity include openness, diversity, partial autonomy and interconnectedness of agents, lack of centralized control, and emergence. The complex systems' components interlinking and interacting with its environment exhibit considerable interdependence.

Since emergence, the largely unexpected manifestations of observable properties, is a prominent characteristic of complex systems, we must recognize the likely existence of heightened degrees of process uncertainties. In attempting to handle these uncertainties, one is advised to bring healthy doses of humility to bear [1], and to try to instil a spirit of flexibility in those involved in service production and management operations, and information, monetary, and expertise exchanges, at all levels of our healthcare institutions or organizations.

Healthcare service development involves organizing people to act and interact effectively not only in person but also in utilizing IT. Importantly, most everyone involved in such an effort needs to adopt an open and constructive attitude of respecting and deeply listening to others with the hopes of increasing the collective understanding of the underlying realities of healthcare problems while creating appropriate bases for possible solutions. During such discussions, the group should also include attempts to clarify the boundary of the complex system to be improved, hopefully with consensus. Restricting the boundary too much may omit key elements of the problem, while expanding the boundary too much may make viable solutions nearly impossible. As Einstein has challenged us, make the problem as simple as possible but no simpler!

\section{ELABORATION}

From practical and ethical viewpoints healthcare service development is a global challenge. We now, however, turn to focus on the status quo of healthcare services in one of the world's most affluent nations, the US. As discussed thoroughly in [14] the US healthcare system may be terminally broken unless some more widespread and additional drastic measures are taken to further improve and correct the current situation. It is generally accepted that several European healthcare systems cost about half of what the US is expending under our current 
"fee-for-service" system. And one can argue whether US healthcare results are any more effective than those of the more socialized European systems, even discounting the large number of US residents that don't have access to healthcare. Here we will focus on the US.

\subsection{Let's Somehow Change the Status Quo for the Better}

According to [14] approximately a quarter of a million people die every year from the shortcomings of the US healthcare system. There are misdiagnoses, inappropriate treatments, mistakes in delivering the proper medication and other remedies, etc.

Medical information and health records are still not being pervasively shared between or among hospitals. This resistance is due largely to protecting hospital "businesses" by jealously hiding medical information that may be useful to others, often citing the need to protect patients' privacy. Incompatibility of data processing and interoperability equipment, software, and procedures, for example, is also a paramount issue [15].

The prevailing fee-for-service dominated practices tend to reward more care rather than the better care. More than $90 \%$ of the doctors get paid that way, and almost $80 \%$ say they don't want to change from that system. Approximately $\$ 1$ trillion is wasted on treatments that patients do not really need.

Almost everyone in the system is charging "what the market will bear." In 2007, for example, lobbyists spent about $\$ 150$ million to avoid changing that paradigm. They dominate in market control. There are instances of monopolistic behaviors which exacerbate cost increases such as with the recent epinephrine auto-injector EpiPen [16] fiasco.

Hospitals are investing in specialty care because that's good for the "bottom line." It appears that nothing will change the current set of incentives. Most players appear to be content with current practices and will likely resist any systemic attempts to provide more attractive cost saving measures.

Further population growth may lead to greater difficulties in funding proper education to help embolden future generations to counterbalance the current trend toward "Trumpism" in the US and reactionary nationalism in several other countries, including France, Germany, and the UK, for example. If the lower and middle classes, as well as women in general, continue to earn relatively less on almost any comparison scale, as robots take over more and more jobs, putting people out of work and making normal jobs and reeducation more difficult to attain [17], the have-nots will be even more unable to afford sending their children to college, and may become still angrier at the establishment who they believe is ignoring them [18]. All this and more such ilk does not bode well!

Being an affluent nation does not automatically secure healthcare service quality. Organizing quality through use of IT need not be expensive if effectiveness is, as it should be, measured principally as customer (in this case, patient) value. This organizing effort, at a minimum, strategically requires proactive, purposeful, and high-minded networking between the operational healthcare providers and relevant context-forming government institutions.

\subsection{Here Are Some Hopeful Suggestions}

Quantum leaps in achieving better healthcare can only occur through behavioral change, and behaviors will only change with massive transformation of incentives. Most people will not pursue moral imperatives to operate beyond the current status quo of how their performance is measured and sustained without being rewarded somehow for doing so. 
As has already been stated here and elsewhere there is a crying need to do better in sharing health record information. This can easily be done with current technology. What is more difficult is to break down institutional barriers that protect this information, that clearly took some investment to gather, from disclosure without some form of compensation. Hospitals should increase their degree of sharing while expecting those institutions they share with to reciprocate. If/when they do this mutual trust will build to further increase the "win-win situation" for the benefit of patients on both sides. There also must be an effort, which almost goes without saying, that certain personal information about the patients should certainly be withheld to protect their privacy. However, their basic medical data, focused on ailments that are useful for treating others, mainly from a database perspective, are what is critical. Naturally, considerable effort must be expended by the stakeholders to decide just which subset of information should be: (1) withheld, in general; and (2) which should be shared. And it could well be that another plethora of data could be in limbo until it is decided how to generate additional information, and into which of these two bins that subset should be placed.

Much can be done to reduce drug costs. Government subsidies have already been shown to help, particularly with the elderly and other disadvantaged patients. First and foremost, doctors should be more assertive in convincing patients to take the many lower-cost drugs that are just as effective as the more expensive drugs pharmaceutical companies are pushing in their advertising, especially on television (TV). In addition, many name-brand drugs are available in Canada, sometimes at ten percent of the cost of the same drugs in the US. Members of Congress must do better in exhibiting (hopefully, their innate) integrity tangibly in caring more about the health of their constituents than in bending to the temptations of lobbyist donor contributions. Representatives and Senators can be reelected in other ways than by outrageously expensive and offensive TV ads. For example, taking meaningful actions that help keep their constituents healthy can be explained in town hall meetings and the like. To the extent these actions are going in the proper direction, their constituents would likely spread this good news like wildfire via word of mouth and, indeed, via TV interviews once the media "steps up" to support such a trend!

Strengthening alliances among hospital administrators, doctors, nurses, patients, families, etc., can make the difference. Better healthcare practices start locally. It's all about more meaningful action-oriented communication and implementation of better ideas.

Does change, following the dominant deterministic model of management, really need to come from the top, and will this soon even be feasible? The second author happened to have an informal conversation with a local hospital administrator. He refuted our contention that costs would decrease if doctors would keep people healthy rather than just treating the sick. He countered, if someone does not receive healthcare and consequently dies of a heart attack, there are essentially no costs; whereas, if someone seeks consultations for a "bum" knee, say, subsequently undergoes remedial physical therapy, then a cortisone shot, yet needs an eventual knee replacement, that is quite costly.

As in complex systems, more significant experimentation with changes that may yield positive improvements will likely come from the bottom, especially considering the moral imperatives for keeping people healthier. Having governmental cutbacks on education and other social costs to pay for healthcare is not the way to go!

Again, behavior change is the key. More doctors are advocating healthy food as a medicinal remedy. Mandated societal changes can also be quite powerful in achieving heath gains. In Amsterdam, Copenhagen, and other European cities, for instance, there are thousands of 
bicyclists. Sure, there are a few bicyclists in the US but we are still quite wedded to cars and Sport Utility Vehicles (SUVs), and eschew public transportation.

The US spends comparatively much less on social services than several other Western countries. Thus, if one compares total healthcare costs more fairly, perhaps, the US actually spends less than approximately twice as much, as alluded to earlier. Also, again touching US politics, if healthcare costs are considered as a percentage of GNP, this statistic for the Affordable Care Act (ACA) ("Obamacare") has been essentially "flat" [14].

The prevailing trend of training more specialists than general practitioners (GPs) is starting to be reversed. Evidently, new doctors are now largely not going into fee-for-service [14]. Clearly, technology can be better applied to free-up doctors to spend more quality time with their patients, other than the few minutes (on the other side of waiting room, technician prep, and examination room waiting times) currently offered by many specialists.

And there are other significant efforts to move away from the more or less standard fee-forservice. For example, there is "bundling": "Bundled payments are a mechanism to deliver a single payment to providers for all the care an individual receives as treatment for a specific acute event or chronic condition. The benefits to health plans can be substantial, including: market incentives for providers to deliver efficient care and encourage patient compliance, reduced variation in the process and cost of care, and improved care coordination resulting in better quality" [19].

Capitation is another initiative that is being implemented: "Capitation is a fixed amount of money per patient per unit of time paid in advance to the physician for the delivery of health care services. The actual amount of money paid is determined by the ranges of services that are provided, the number of patients involved, and the period of time during which the services are provided" [20]. There are quite a few healthcare examples of doing better. Realistically though, broad, significant changes typically take about four years before they catch on, and ten more years are necessary for them to be more completely instituted. After all, it's a \$3.4T industry [14]. Then there is the big idea of "single payer" which is an anathema to conservatives, generally, especially because "socialist" Bernie Sanders has been one of the strong advocates. If one accepts most of what conservatives would dismiss as untrue propaganda from organizations like Public Citizen [21], there is much that could be said logically and logistically, at least, about how there could be tremendous savings in current administrative and insurance costs by marginalizing the big insurance companies and providing Medicare for all, so the risk pool is appropriately widened to include mostly healthy people. Unfortunately, selling this to the young and healthy, who may have some difficulty contemplating future personal health problems, requires an appeal to what could happen to them as well as their moral sensibilities.

\subsection{Connectivity, Big Data, and Information Technology}

The most basic Internet of Things (IoT) concept is to enable and nurture connectivity among a multitude of autonomous electronic devices for human benefit; this: (1) could simplify our lives; (2) can be financially profitable; (3) offers pathways to professional satisfaction; and (4) presents cybersecurity opportunities. But is this all good? What about potential unintended consequences which may seriously degrade our quality of life? Will the IoT, even if implemented well in the face of formidable technical challenges, really improve our quality of life? We need to ask about and firmly establish the essential roles, cares, and desires of people in this world of automated artefacts. 
This ethical concern can be elaborated briefly, for example, addressing each of the four motivations listed just above ([10] provides more detail.): (1) one automation-related outcome of IoT is that robots are taking over more and more jobs [17]; (2) many organizations care more about unlimited material growth [22] than improving the lot of humankind; (3) individual efforts often tend to divide people rather than bring us together; and (4) security is hampered by trying to protect too much instead of realizing the benefits of information sharing [1].

We are also critical of the BD term since the phenomenon it represents cloaks the true nature of the electronic information that should be embedded in many applications, particularly supply chains. In most services, it is not the vast bulk of hidden data, but real-time, carefully selected data of production value to agents and changing customer value perceptions, that need to be mined. Again, creating strategic connectivity should include elaborating on the role of people in the healthcare production system.

Knowledgeable healthcare stakeholders, after determining what works and what does not, should contact state-of-the-art technologists for help on what IT enablers might be tried. All this depends on sharing information, building trust, and striving for open mindsights, keeping the health and well-being of patients as the ultimate goal. This will more clearly define the system roles of value-producing agents.

An important and fundamental task in any supply chain is suitably integrating its diverse agents. One of the primary goals of recent years is improving the appropriate and worthwhile sharing of medical data. Doing so represents an expression of healthcare supply chain integration. Although IT is capable of this, as already stated, institutional profit barriers tend to hamper its achievement in healthcare services. And again, patients need to share at least their trend-able data to help improve general public health.

Safeguarding caregivers from routine mistakes in treatment is another critical area for improvement. IT should be able to revamp existing checklist processes in a simple, less time-consuming, and more accountable way. ISs of this sort should be designed to relieve doctors, nurses and technicians from time-wasting data-entry and retrieval tasks.

The target logistical IS must capture, store, and communicate to other agents, dynamically, data about resource availabilities and usages. So, when looking to apply the latest medical tool, IT, or IS as part of any healthcare endeavor, examine the pros and cons from this point of view. Important advances in this direction can be made considering very recent work that leverages cloud-based information sharing to improve hospital supply chain performance [23].

Exorbitant prices are imposed by drug companies and their lobbyists [24]. Again, as we have stated, patients need to largely ignore propaganda, about medicines they hear about on $\mathrm{TV}$, for example, and doctors need to prescribe the much less expensive but effective alternative medicines.

In the provision of healthcare services, the questions of ethics should focus mainly on the well-being of the patients which includes, of course, lowering costs. This evidently includes eliminating, or at least mitigating, fee-for-service specialties. In terminal cases is not it better to provide services that induce feelings of comfort and contentment rather than more pain and suffering [25]?

Clearly, different incentives for all stakeholders are needed. Doctors should be rewarded for keeping people healthy. Hospitals should no longer be run as profit-making businesses. Insurance and pharmaceutical companies should operate in the public interest; politicians need insulation from lobbyists. We probably need to approach healthcare that people can afford, no matter where in the world they reside.

Additional guidance on these topics and managing supply chains can be found in [10]. 


\section{SPECIFIC EXAMPLES}

Reference [6] advocates a case study approach in learning more about complex systems and furthering complex systems engineering, and contains 23 case studies, one (Chapter 7) having much to do with healthcare. A case study template that can be used as a guideline in reporting on case studies of complex healthcare systems can be found in Chapter 3 .

Reference [10] is focused on three (actually mini-) case studies central to the healthcare supply chain. In the next subsection, we summarize these as typical examples of what healthcare professionals face in their daily work lives. Together these cases provide different windows into issues regarding integration and information connectivity in healthcare service supply chains.

\subsection{Case Study Overviews}

The first case provides a narrative of commodity flow at one of the state-owned hospitals in Thailand. To understand the logistics flow of medical commodities in the hospital, one type of frequently-used medicine was selected and studied within its supply chain. A focus group, reviewing and assessing the results in depth, reached consensus on a promising way forward.

Managing material flows is a core feature of logistics. Here the flow of goods is a supporting but not core element, given that we are studying a services industry. One of the key aspects of this flow is the overstocking problem. One factor that contributes to the exacerbation of the problem are the natural and persistent time delays that may occur between steps in the overall logistical supply chain process. Possible mitigations to this phenomenon would be to endeavor to apply IT more effectively to not only reduce the manual labor of the participants but also to speed up the process, thereby reducing the human errors as well as the time delays.

The second case concerned waiting lists, specifically the treatment of a psychiatric patient focusing on consequences of unexpected and poorly informed changes of that type of flow. This involved an authentic incident that occurred in an outpatient psychiatric clinic in a Norwegian Hospital Trust, and represents customary priority setting procedures. Again, much was learned that benefited an approach to future improvements.

This case highlighted a problem of "falling through the cracks." A doctor scheduled to treat a patient got sick beforehand, an event that was unanticipated by the system. Thus, a seeming paradox ensued, a patient was recorded as finishing treatment in the general waiting list, despite still being ill, because the status of the patient recorded in a local waiting list was not taken into consideration as data in the general waiting list.

The third case addressed the extent to which effective communication exists in emergency situations, in particular, the standardized organization of information flow related to moving patients between hospitals and home care facilities in Norway. This took place when GPs and nurses in a home care system in a Norwegian municipality described how they communicate with the staff at a state-owned hospital. Opportunities for improving IT supported improvements were illuminated, for example.

The hospital physicians, GPs, and home care nurses usually communicate by electronic messaging. Yet these messages and even phone calls often used for clarification, do not accurately impart and capture all the relevant information and knowledge required. In addition, the nurses perceived an increased risk of misunderstanding if there were no inter-professional/personal contacts or meetings. Therefore, both nurses and physicians advocated for the importance of working together in person which naturally leads to further clarifications as well as new information and knowledge. 
Reading more about these (mini-)cases and the issues they raise, consider how they could be expanded into full-fledged case studies.

\subsection{Case Study Summary}

There is a dearth of good ways to clarify responsibilities among the different professions involved. Communication is often cited as the most important factor for inter-professional cooperation, both by the nurses and even more by the GPs. However, electronic communication cannot substitute for the positive effects of meeting each other face-to-face to experience the often subtle but invaluable cues.

In a complex system, management must consider how to handle processes where components are continually in flux regarding not only the more easily measurable time, place, and form features, but also how they are interconnected (pooled) as well as how they are perceived. In this supply-chain, viewed as a complex system, logistics is an emergent process because the services and how they are evaluated over time are both transformed in each stage of production. Therefore, co-creation in healthcare logistics services is important. The role of IS here is to support process emergence. This involves data registration, informing each other about this emergent process's properties, and thereby supporting agency in sensemaking and managing this process in real time. Connectivity is a key attribute of such an IS, leveraging computer-human interfaces expressed as streamlined and visualization techniques that interconnect different supply chain agents. This means seeking and implementing an ethically viable potential for automating agency in the healthcare service supply chain where a large number of agents can interact in a non-deterministic way.

\section{SUMMARY}

Healthcare logistics is suggested as a complex system to operate and manage healthcare services in any economy regardless of its stage of development. This is particularly because human beings, viz., patients, physicians, nurses, suppliers, and administrators are centrally involved in providing and experiencing a variety of services and remedial treatments. Being predominately reciprocally interdependent, interaction in healthcare systems is reliant on intense exchanges to mutually adjust the relationships between patients and healthcare service providers. The pressing question regards the quality of this interaction, especially how long it takes to treat, and hopefully cure, the patient.

Existing policies and procedures are oriented mainly toward helping sick people to get well instead of instituting incentives and rewards for keeping people healthy as the higher priority. Admittedly, migrating healthcare more toward the latter mindsight is largely in the domain of government legislative and institutional executive actions and beyond the scope of logistical supply chain improvements. But healthcare providers already have the power to take a more holistic approach in establishing improved methodologies that can facilitate, along with carefully selected IS technologies, more effective interpersonal communication and networked interactions among all parties and elements of this complex system. That's what has been emphasized and explored here.

According to the literature and direct observations of existing practices, most healthcare services are well-intentioned and at least partially effective. However, it is also clear from interviews and analysis that systemic and systematic flaws significantly hinder higher quality healthcare, particularly in situations where time is a critical factor, e.g., in emergencies. Sure, one might prevent many emergencies by keeping people healthier but that is not the way the 
system operates, as we have outlined in Subsections 2.2 and 3.1. So, taking a broader and more humanistic view of the logistical supply problem, leveraging pooled as well as reciprocal interactions, emphasizing additional attention to contingencies, and better applying IT are among the advocated principles to help caregivers deliver better services, their primary function, while alleviating their secondary administrative burdens.

Since there is not yet a well-established and accepted theory that thoroughly informs healthcare service supply chains, case studies are of paramount importance in creating desirable outcomes. We really would like a better understanding of what works-and what doesn't - in practice, and good case studies can usefully illuminate many issues. Three subcases, the: (1) logistical flow of commodities at a hospital; (2) waiting list paradox; and (3) emergency flow and inter-professional communication about patients moving between hospital and home care, have been described and analyzed [10]. These should help set the stage for additional examples and more in-depth case studies in future work.

\section{ACKNOWLEDGEMENTS}

The case narratives provided in Section 4 are based on work-in-progress, where Ingunn Mundal and Trude F. Eines of Molde University College in Norway, and Duangpung Kritchanchai of Mahilol University in Thailand, provided the narrative based on their investigations. This work, as detailed in [10] is currently in the process of being published in an academic journal. The authors also thank Chad Walker of Analog Devices for alerting us to the cogent, stimulating, and entertaining Intelligence Squared video debate [14].

\section{REFERENCES}

[1] White, B.E., A Complex Adaptive Systems Engineering (CASE) Methodology: The Ten-Year Update, Presented at IEEE Systems Conference, Orlando, FL, 18-21 April 2016.

[2] Bar-Yam, Y., Making Things Work: Solving Complex Problems in a Complex World, 1st Edition. NECSI Knowledge Press, 2005.

[3] Kay, J.J., An Introduction to Systems Thinking. The Ecosystem Approach: Complexity, Uncertainty, and Managing for Sustainability, eds. D. Waltner-Toews, J.J. Kay \& N-M.E. Lister, Columbia University Press: New York, pp. 3-13, 2008.

[4] Kay, J.J., Framing the Situation: Developing a System Description. The Ecosystem Approach-Complexity, Uncertainty, and Managing for Sustainability, eds. D. WaltnerToews, J.J. Kay, \& N-M.E. Lister, Columbia University Press: New York, pp. 16-34, 2008.

[5] Funtowicz, S. \& Ravetz, J., Beyond Complex Systems: Emergent Complexity and Social Solidarity. The Ecosystem Approach - Complexity, Uncertainty, and Managing for Sustainability, eds. D. Waltner-Toews, J.J. Kay \& N-M.E. Lister, Columbia University Press: New York, pp. 309-321, 2008.

[6] Gorod, A., White, B.E., Ireland, V., Gandhi, S.J. \& Sauser, B.J., Case Studies in System of Systems, Enterprise Systems, and Complex Systems Engineering, CRC Press, Taylor \& Francis Group: Boca Raton, FL, 2015.

[7] Norman, D.O. \& Kuras, M.L., Engineering Complex Systems. Complex Engineered Systems: Science Meets Technology, eds. D. Braha, A. Minai, Y. Bar-Yam, Springer: New England Complex Systems Institute, Cambridge, MA, 2006.

[8] White, B.E., On leadership in the complex adaptive systems engineering of enterprise transformation. Journal of Enterprise Transformation, 5(3), pp. 192-217, 2015.

http://dx.doi.org/10.1080/19488289.2015.1056450 
[9] Gilbertson, R., Tanju, B. \& Eveleigh, T.J., A complexity based heuristic decision analysis model to recommend systems engineering domain. IEEE Engineering Management Review, 45(3), pp. 64-81, 2017.

https://doi.org/10.1109/emr.2017.2734358

[10] Engleseth, P., White, B.E., Mundal, I., Eines, T.F. \& Kritchanchai, D., Healthcare logistics as a complex system. Production Planning \& Control, In review.

[11] Hawn, G. (with Holden, W.), 10 Mindful Minutes, Penguin Books: New York, p. xiv, 2011.

[12] Ashby, W.R., Requisite variety and implications for control of complex systems. Cybernetica, 1(2), pp. 83-99, 1958.

[13] Rzevski, G. \& Skobelev, P., Managing Complexity, WIT Press: Southhampton, UK, 2014.

[14] Proposition, The U.S. Health Care System is Terminally Broken, Intelligence Squared Debates, available at https://www.intelligencesquaredus.org/debates/us-health-caresystem-terminally-broken (accessed 21 October 2017)

[15] A Robust Health Data Infrastructure, prepared for: Agency for Healthcare Research and Quality, 540 Gaither Road Rockville, MD, 2014, available at www.ahrq.gov (Contract No. JSR-13-700, Prepared by: JASON, The MITRE Corporation, 7515 Colshire Drive McLean, VA 22102-750, AHRQ Publication No. 14-0041-EF, April 2014.)

[16] Epipen, 2017, available at: https://www.reuters.com/article/us-health-epipen-costs/ another-look-at-the-surge-in-epipen-costs-idUSKBN16Y24O

[17] White, B.E., Fathoming the future of artificially intelligent robots. International Journal of Design \& Nature and Ecodynamics, 13(1), pp. 1-15, 2018. https://doi.org/10.2495/dne-v13-n1-1-15

[18] Bageant, J.L., Deer Hunting with Jesus. Three Rivers Press: New York, 2007.

[19] Bundling, 2017, available at https://truvenhealth.com/portals/0/assets/HP_11512_0812_ BundledPayments_SS_WEB.pdf

[20] Capitation, 2017, available at https://www.acponline.org/about-acp/about-internalmedicine/career-paths/residency-career-counseling/guidance/understanding-capitation

[21] Public Citizen, mass mailing material, 1600 20th Street, NW, Washington, D.C., 2017, available at www.citizen.org

[22] White, B.E., Let's Do Better in Limiting Material Growth to Conserve Our Earth's Resources, Conference on Systems Engineering Research (CSER) 2012, St. Louis, MO, 19-22 March 2012.

[23] Kochan, C.G., Nowicki, D.R., Sauser, B. \& Randall, W.S., Impact of cloud-based information sharing on hospital supply chain performance: a system dynamics framework. International Journal of Production Economics, 195, pp. 168-185, 2017. https://doi.org/10.1016/j.ijpe.2017.10.008

[24] Abramson, J., Overdosed America: The Broken Promise of American Medicine, 3rd edn, Harper-Collins: New York, 2004.

[25] Gawande, A., Being Mortal: Medicine and What Matters in the End, Henry Holt: New York, 2004. 